\section{Molecular Characteristics of a Series of Clinical Isolates of Drug-Resistant Acinetobacter baumannii ST219 Strain: The Implications of a Sequence Analysis of the blaoxa-51-like}

\begin{abstract}
Kazuo Umezawa ${ }^{1}$, Satomi Asai ${ }^{2 *}$, Hideo Iwashita ${ }^{3}$, Yuki Minakawa ${ }^{4}$, Maya Ohashi ${ }^{4}$, Toshio Ohshima ${ }^{4}$, Hideki Hayashi ${ }^{5}$, Mari Matsui ${ }^{6}$, Sadaki Inokuchi ${ }^{1}$ and Hayato Miyachi ${ }^{2}$
\end{abstract}

${ }^{1}$ Department of Emergency and Critical Care Medicine, Tokai University School of Medicine, Isehara, Kanagawa, Japan

${ }^{2}$ Department of Laboratory Medicine, Tokai University School of Medicine, Isehara, Kanagawa, Japan

${ }^{3}$ Department of Adult Dentistry, Yokohama Clinic, Kanagawa Dental University, Yokohama, Kanagawa, Japan

${ }^{4}$ Division of Infection Control, Tokai University Hospital, Isehara, Kanagawa, Japan

${ }^{5}$ The Support Center for Medical Research and Education of Tokai University, Tokai University School of Medicine, Isehara, Kanagawa, Japan

${ }^{6}$ Department of Bacteriology II, National Institute of Infectious Diseases, Tokyo, Japan

\begin{abstract}
The drug-resistant Acinetobacter baumannii ST219 strain was sporadically isolated in the emergency intensive care unit of Tokai University Hospital in 2014 after an outbreak in 2013. The isolates were identical in their antimicrobial susceptibility pattern, the fingerprint pattern of rep-PCR, and the molecular properties including mutations in drug-resistant genes and decreased expression of the efflux pump and the outer membrane porin genes, but they were found to possess different sequence types based on the findings of multilocus sequence typing: ST208 and 219. The analysis of the blaoxa-51-like sequences showed that all were bla ${ }_{\text {OxA-66. }}$. Given these findings, all of the isolates were considered to be subclones derived from the same strain. A sequence analysis of the blaoxA-51-like of $A$. baumannii would therefore be useful for investigating the relationship of nosocomial infections.
\end{abstract}

*Corresponding author: Satomi Asai, Department of Laboratory Medicine Tokai University School of Medicine, Shimo-kasuya 143, Isehara, Kanagawa, Japan. Tel: +81-463-93-1121; E-mail: sa@is.icc.u-tokai.ac.jp

Citation: Umezawa K, Asai S, Iwashita H, Minakawa Y, Ohashi M, et al. (2016) Molecular Characteristics of a Series of Clinical Isolates of Drug-Resistant Acinetobacter baumannii ST219 Strain: The Implications of a Sequence Analysis

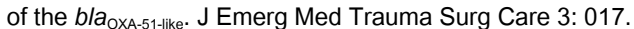

Received: January 28, 2016; Accepted: June 17, 2016; Published: June 30, 2016
Keywords: Acinetobacter baumannii; Drug resistance; Outer membrane porin; OXA-type $\beta$ lactamase; ST219

\section{Introduction}

Acinetobacter baumannii is a strictly aerobic, non-fermenting, non-fastidious, non-motile, catalase-positive, oxidase-negative, gram-negative bacteria that is known to be a major pathogen of nosocomial infection in immunocompromised patients [1]. Outbreaks of antimicrobial-resistant A. baumannii have become a major clinical problem [1-3]. All strains of A. baumannii possessed a chromosomally encoded $b l a_{\text {OXA-51-likee }}$ some of which provided resistance to carbapenems when the molecular milieu around the gene promoted its expression, such as ISAba1 [4]. The $b l a_{\text {OXA-51-like }}$ has been reported to have sequence variations with over 40 variants $[5,6]$.

We previously reported an outbreak of an amikacin- and ciprofloxacin-resistant $A$. baumannii sequence type (ST)219 strain that affected 15 patients in the emergency intensive care unit of Tokai University Hospital from September to October in 2013 [3]. Intensive control measures were implemented, including the replacement of the water supply system, which was considered to be a bacterial reservoir, and thus could successfully control the outbreak. However, sporadic cases of A. baumannii, with an identical pattern of antimicrobial susceptibility, were subsequently detected in 2014 . The present study was undertaken to elucidate the molecular characteristics for antimicrobial resistance and typing for epidemiology in drug resistant (DR) A. baumannii in 2014.

\section{Materials and Methods}

After an outbreak of DR-A. baumannii that affected 15 patients from September to October 2013 [3], DR-A. baumannii was sporadically detected in sputum specimens from six patients from January to October 2014 (TS-A. baumannii-2014-1 to -6). These patients were treated in the emergency intensive care unit ( 57 beds, including 3 beds in the severe burn care unit) of Tokai University Hospital (total 804 beds) for serious burns, traffic injuries, or cerebral hemorrhaging. Routine microbial examinations were performed on a weekly basis in clinical specimens from the patients' sputum, urine (via catheter), venous blood and wounds, among other bodily fluids. One drug-susceptible A. baumannii (TS-A. baumannii-2014-7) was used as a control for quantitative RT-PCR (qRT-PCR).

Antimicrobial susceptibility testing, and screening of carbapenemase, MBL, ESBL and AmpC

Bacterial identification and antimicrobial sensitivity tests were performed using the MiroScan WalkAway 96 Plus kit (Beckman Coulter, Inc., CA, USA) in accordance with the CLSI 2010 guidelines [7] for Imipenem (IPM), Meropenem (MEPM), Piperacillin (PIPC), Ceftazidime (CAZ), Cefcapene (CFPM), Sulbactam/Cefoperazone (S/C), Aztreonam (AZT), Cefozopran (CZOP), Gentamicin (GM), Tobramycin (TOB), Amikacin (AMK), Levofloxacin (LVFX), Ciprofloxacin (CPFX), Minocycline (MINO), Fosfomycin (FOM) and Sulfamethoxazole-Trimethoprim (ST). The criteria for multiple drug-resistant A. baumannii was resistance to IPM (MIC $>16 \mu \mathrm{g} / \mathrm{mL}$ ), AMK 
Citation: Umezawa K, Asai S, Iwashita H, Minakawa Y, Ohashi M, et al. (2016) Molecular Characteristics of a Series of Clinical Isolates of Drug-Resistant Acineto-

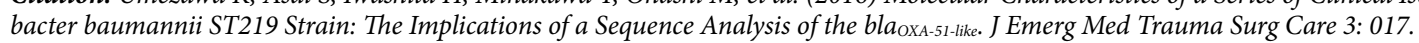

$(\mathrm{MIC}>32 \mu \mathrm{g} / \mathrm{mL})$ and CPFX $(\mathrm{MIC}>4 \mu \mathrm{g} / \mathrm{mL})$, and DR-A. baumannii was defined as resistance to at least 2 of the drugs, according to the Japanese National Guideline concerning the prevention of infections and medical care for patients with infections.

A. baumannii isolates were screened for the production of metallo- $\beta$-lactamase (MBL) by Double-Disk Synergy Tests (DDST) (Eiken Chemical Co., Ltd. Tokyo, Japan) using extended-spectrum $\beta$ lactamase (ESBL), in accordance with the CLSI 2010. The isolates were screened for the production of AmpC using 3-aminophenylboronic acid monohydrate (Kanto Chemical Co., Inc., Tokyo, Japan).

\section{Molecular typing}

The first detected isolate of $A$. baumannii in each patient was used for the following molecular characterizations. DNA was extracted using the QIAamp DNA Mini Kit (QIAGEN GmbH, Hilden, Germany). A. baumannii isolates were screened for gene homology by the repetitive-sequence-based-polymerase chain reaction (rep-PCR), as described previously [8]. The BOX-PCR primer was used (5'-CTA CGG CAA GGC GAC GCT GAC G-3'). The temperature profiles were as follows: initial denaturation at $95^{\circ} \mathrm{C}$ for $3 \mathrm{~min}, 30$ cycles of denaturation at $90^{\circ} \mathrm{C}$ for $30 \mathrm{~s}$, annealing at $32^{\circ} \mathrm{C}$ for $1 \mathrm{~min}$, extension at $65^{\circ} \mathrm{C}$ for $8 \mathrm{~min}$, and a final extension at $65^{\circ} \mathrm{C}$ for $16 \mathrm{~min}$.

Multilocus sequence typing (MLST) was performed as described previously [9]. The MLST sequences were uploaded into the A. baumannii MLST sequence type database (http://pubmlst.org/ abaumannii/) to determine the alleles and sequence types (ST). Clonal complexes (CCs) were assigned using the eBURST V3 software program (http://eburst.mlst.net/v3/) and defined as single locus variants (SLVs) and double locus variants (DLVs). The annealing temperature of the PCR amplification used in this study was $55^{\circ} \mathrm{C}$ for gltA, gyrB, recA and $c p n 60$, and $50^{\circ} \mathrm{C}$ for $g d h B, g p i$ and $r p o D$. The amplification products were purified with a QIAGEN DNA purification kit (QIAGEN GmbH). The DNA sequencing was performed using an ABI3500xL Genetic Analyzer (Applied Biosystems, Life Technologies Japan Ltd., Tokyo, Japan).

\section{PCR assay for $\beta$-lactamase and arm $A$}

The resistance genes were examined via a multiplex PCR for $b l a_{\text {OXA-23-like }} b l a_{\text {OXA-24-like }} b l a_{\text {OXA-51-like }} b l a_{\text {OXA-58-like }}$ and ISAbal genes, as described previously [2,10-12]. The amplification of the $b l a_{\text {OXA-51-like }}$ genes was performed as described previously [6,11]. The armA gene, which encodes 16S rRNA methylases and confers high resistance to aminoglycosides (AGs), was screened by PCR using primers as described previously [13].

\section{Sequencing of OXA-type $\beta$-lactamase, and $g y r A$, and parC}

Sequencing of OXA-type $\beta$-lactamase was performed as described previously [14]. The quinolone resistance-determining regions (QRDRs) of $g y r A$ and parC were amplified and analyzed as described previously [14,15]. The DNA sequencing of the amplified DNA products was performed using an ABI3500xL Genetic Analyzer (Applied Biosystems).

\section{Quantitative RT-PCR (qRT-PCR)}

The RNA templates were extracted using the RNeasey ${ }^{\circledR}$ Mini Kit (QIAGEN GmbH). The expression of three different resistance-nodulation-division (RND) family pump-encoding genes (adeB, adeG, $a d e J)$ and two outer membrane porin-encoding genes (oprD and carO) were analyzed by quantitative RT-PCR using the StepOnePlus ${ }^{\mathrm{TM}}$
Real-Time PCR System (Applied Biosystems) [2,16-18]. The three previously characterized genes adeB, ade $G$ and adeJ encoded the RND pumps in the adeABC, adeFG and adeIJK operons, respectively. The housekeeping gene 16S rRNA was used as a control [19-21]. Reactions $(20 \mu \mathrm{L})$ were set up using $400 \mathrm{nM}$ primers and $2 \mu \mathrm{L}$ of the cDNA template (diluted 1:10) with SYBR ${ }^{\otimes}$ Premix Ex Taq ${ }^{\text {TM }}$ II (Tli RNase H Plus) and ROX plus (Takara Bio Inc., Shiga, Japan).

The data were analyzed using the StepOne ${ }^{\mathrm{TM}}$ software program. The expression of each target gene was normalized based on the level of the 16S rRNA mRNA gene and was expressed as a relative rate compared to that in the susceptible isolate of each pair. The expression of drug-susceptible A. baumannii (TS-A. baumannii-2014-7) was set as 1.0 [2]. The experiments were conducted at least three times independently, and all of the reactions were performed in triplicate.

\section{Results}

\section{Antimicrobial susceptibility testing, and screening of carbapenemase, MBL, ESBL and AmpC}

The antimicrobial susceptibility patterns of 6 isolates of A. baumannii in 2014 (TS-A. baumannii-2014-1 to -6) was shown in Table 1. They were resistant to PIPC, CAZ, CFPM, S/C, AZT, CZOP, GM, TOB, AMK, LVFX, CPFX, MINO, FOM and ST. They were negative for MBL, ESBL and AmpC on DDSTs.

\section{Molecular typing}

The molecular genotyping of isolates by a MLST analysis revealed a sequence type of ST219 for TS-A. baumannii-2014-1, -3, -4, -5 and -6 (gltA, gyrB, gdhB, recA, cpn60, gpi, rpoD: 1, 3, 3, 2, 2, 101, 3) and ST208 for TS-A. baumannii-2014-2 (gltA, gyrB, gdhB, recA, cpn60, gpi, rpoD: 1, 3, 3, 2, 2, 97 and 3) (Table 2). The difference in the MLST sequence between ST208 and ST219 was confirmed to be a single gpi base by sequencing the PCR product (data not shown). TS-A. baumannii-2014-7 showed an unknown sequence type (gltA, gyrB, gdhB, recA, cpn60, gpi, rpoD: 15, 48, 58, 42, 36, 54, 41). All of the isolates (TS- $A$. baumannii-2014-1 to -6,) except for TS-A. baumannii-2014-7 shared the same fingerprint pattern in the rep-PCR (Figure 1).

\section{Characteristics of the regions involved in antimicrobial resistance}

The results of the gene analysis are shown in Table 2. Among the OXA- $\beta$-lactamases, $b l a_{\text {OXA-51-like }}$ was detected in all isolates, together with ISAbe1 in TS-A. baumannii-2014-1 to -6 . The investigation of the $b l a_{\text {OXA-51-like }}$ sequences resulted that TS- $A$. baumannii-2014-1 to -6 carried $b{ } a_{\text {OXA-66 }}$. They showed the expression of armA for AGs resistance. PCR and sequencing of TS- $A$. baumannii-2014-1 to -6 revealed a point mutation of gyrA (83-Serine to Leucine) and parC (80-Serine to Leucine) for fluoroquinolones (FQs) resistance. The findings for the TS- $A$. baumannii-2014-1 and -2 as the representative strains from each group with the same susceptibility pattern were shown in Table 3. The results of qRT-PCR showed decreased expression of efflux pump (adeB, ade $G$ and $a d e J$ ) and outer membrane porin-encoding genes (oprD and carO).

\section{Discussion}

Clinical isolates of an outbreak in 2013 and subsequent sporadic detection in 2014 of DR-A. baumannii in the emergency intensive care unit of Tokai University Hospital showed resistance to a broad spectrum of antimicrobials except for carbapenems, and were found to have $b l a_{\text {OXA-51-like }}$ carrying $b l a_{\text {OXA-66 }}$ with ISAba1, alterations 
Citation: Umezawa K, Asai S, Iwashita H, Minakawa Y, Ohashi M, et al. (2016) Molecular Characteristics of a Series of Clinical Isolates of Drug-Resistant Acinetobacter baumannii ST219 Strain: The Implications of a Sequence Analysis of the bla OXA-51-like. J Emerg Med Trauma Surg Care 3: 017.

- Page 3 of $4 \cdot$

\begin{tabular}{|c|c|c|c|c|c|c|c|c|c|c|c|c|c|c|c|c|}
\hline \multirow[t]{3}{*}{ Strain } & \multicolumn{16}{|c|}{ MIC ( $\mu \mathrm{g} / \mathrm{mL})$} \\
\hline & \multicolumn{8}{|c|}{$\beta$-Lactams } & \multicolumn{3}{|l|}{ AGs } & \multicolumn{2}{|l|}{ FQs } & \multicolumn{3}{|c|}{ Other agents } \\
\hline & IPM & MEPM & PIPC & CAZ & CFPM & $\mathrm{s} / \mathrm{C}$ & AZT & CZOP & GM & ТОВ & AMK & LVFX & CPFX & MINO & FOM & ST \\
\hline $\begin{array}{l}\text { TS-A. bauman- } \\
\text { nii-2014 -1,-2,- } \\
\text { 3,-4,-5,-6 }\end{array}$ & $\leq 1(\mathrm{~S})$ & $\leq 1(\mathrm{~S})$ & $>64(\mathrm{R})$ & $>16(\mathrm{R})$ & $16(\mathrm{R})$ & $<16(S)$ & $>16(R)$ & $16(\mathrm{R})$ & $>8(\mathrm{R})$ & $>8(\mathrm{R})$ & $>32(\mathrm{R})$ & $>4(\mathrm{R})$ & $>2(\mathrm{R})$ & $4(\mathrm{~S})$ & $>16(R)$ & $>2(R)$ \\
\hline $\begin{array}{l}\text { TS-A. bauman- } \\
\text { nii-2014 -7 }\end{array}$ & $\leq 1(\mathrm{~S})$ & $\leq 1(\mathrm{~S})$ & $\leq 8(S)$ & $4(S)$ & $<4(S)$ & $<16(S)$ & $8(\mathrm{~S})$ & $8(\mathrm{~S})$ & $2(S)$ & $2(S)$ & $8(\mathrm{~S})$ & $\leq 0.5(\mathrm{~S})$ & $1(\mathrm{~S})$ & $\leq 2(\mathrm{~S})$ & $<16(\mathrm{~S})$ & $\leq 2(\mathrm{~S})$ \\
\hline
\end{tabular}

Table 1: The antimicrobial susceptibility pattern of the clinical isolates of Acinetobacter baumannii.

MIC- minimum inhibitory concentration; AGs- aminoglycosides; FQs- fluoroquinolones; IPM- imipenem; MEPM- meropenem; PIPC- piperacillin; CAZ- ceftazidime; CFPM- cefepime; S/C- sulbactam/cefoperazone; AZT- aztreonam; CZOP- cefozopran; GM- gentamicin; TOB- tobramycin; AMK- amikacin; LVFX- levofloxacin; CPFX- ciprofloxacin; MINO- minocycline; FOM- fosfomycin; ST- sulfamethoxazole/ trimethoprim; R- resistant; S- susceptible.

\begin{tabular}{|c|c|c|c|}
\hline & $\begin{array}{c}\text { TS- } A \text {. baumannii-2014 } \\
-1,-3,-4,-5,-6\end{array}$ & $\begin{array}{c}\text { TS-A. baumannii-2014 } \\
-2\end{array}$ & $\begin{array}{c}\text { TS-A. baumannii-2014 } \\
-7\end{array}$ \\
\hline Sequence type & 219 & 208 & unknown* \\
\hline bla $a_{\text {OXA-24-like }}$ & - & - & - \\
\hline$b / a_{\text {OXA-58-like }}$ & - & - & - \\
\hline$b / a_{\text {OXA-51-like }}$ & + & + & + \\
\hline$b l a_{\text {OXA-66 }}$ & + & + & - \\
\hline $\operatorname{arm} A$ & + & + & - \\
\hline \multicolumn{4}{|l|}{ QRDRs } \\
\hline gyrA & $83 \mathrm{Ser} \rightarrow$ Leu & $83 \mathrm{Ser} \rightarrow$ Leu & 83 Ser \\
\hline parc & $80 \mathrm{Ser} \rightarrow$ Leu & $80 \mathrm{Ser} \rightarrow$ Leu & 80 Ser \\
\hline
\end{tabular}

Table 2: The characteristics of antimicrobial resistance in the clinical isolates of Acinetobacter baumannii.

QRDRs- quinolone resistance-determining regions.

* TS-A. baumannii-2014-7 showed an unknown sequence type (gltA, gyrB, gdhB, recA, cpn60, gpi, rpoD: 15, 48, 58, 42, 36, 54, 41).

\begin{tabular}{|c|c|c|c|}
\hline & TS-A. baumannii-2014-1 & TS-A. baumannii-2014-2 & TS-A. baumannii-2014-7 \\
\hline \multicolumn{4}{|c|}{ Efflux pump expression } \\
\hline$a b e B$ & 0.34 & 0.49 & 1.00 \\
\hline abeJ & 0.69 & 0.55 & 1.00 \\
\hline \multicolumn{4}{|c|}{ Outer membrane porin expression } \\
\hline oprD & 0.17 & 0.01 & 1.00 \\
\hline carO & 0.02 & 0.33 & 1.00 \\
\hline
\end{tabular}

Table 3: Relative expression of efflux pumps and outer membrane porin in clinical isolates Acinetobacter baumannii by quantitative RT-PCR.

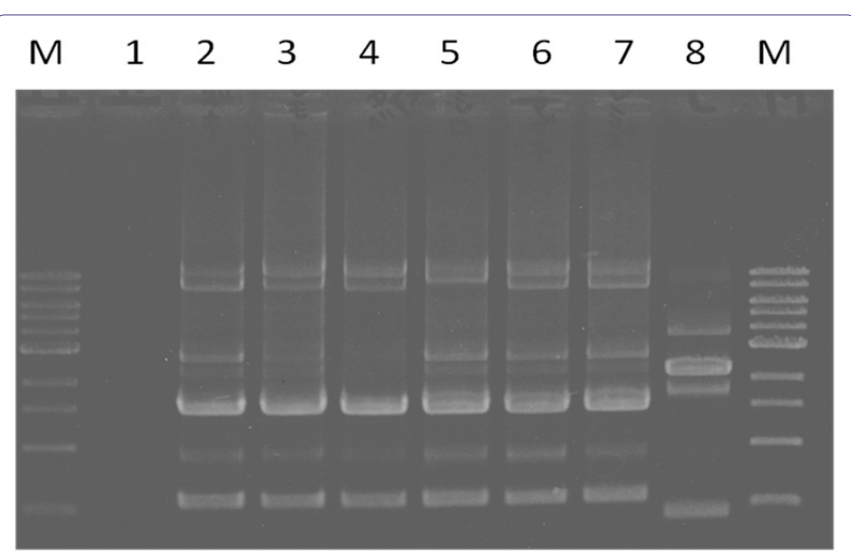

Figure 1: The results of the rep-PCR in clinical isolates of $A$. baumannii.

Lanes 1 was negative control of the PCR. Lanes 2 to 7 indicate drug resistant- $A$. baumannii (TS- $A$. baumannii-2014-1 to -6 ), and lane 8 is drug susceptible- $A$. baumannii (TS- $A$. baumannii-2014-7), respectively. Lane $\mathrm{M}$ is a marker. TS- $A$. baumannii-2014-1 to -6 showed an identical pattern with a homologous rate of identity $>97 \%$. TS-A. baumannii-2014-7 had different fingerprint pattern (less than $70 \%$ similarity, respectively). of genes responsible for AGs and FQs, and decreased expression of the efflux pump and outer membrane porin-encoding genes. These findings suggested that these isolates of $A$. baumannii shared a molecular basis for the same susceptibility pattern to antimicrobials. These findings are compatible with collateral susceptibility to carbapenem and consistent with a previous report indicating that the combination of OXA-type $\beta$ lactamases with ISAbal and deficiency of outer membrane porins deficiency alone does not confer carbapenem resistance, and that overexpression of the efflux pumps may be necessary $[2,12,22]$.

Isolates of TS-A. baumannii-2014-1 to -6 showed identical molecular characteristics, such as the fingerprint pattern, OXA type, drug resistant genes and expression of efflux pumps. A. baumannii can survive for long-term periods of time in the hospital environment, causing sporadic and endemic infection $[2,3,23]$. We experienced an outbreak twice in the past in the emergency intensive care unit and burn unit of our University Hospital. DR-A. baumannii ST208 was involved in an outbreak in 2011, where the air fluidity bed was identified as a reservoir. An outbreak of DR-A. baumannii in 2013 was detected from the water systems including hands-free automatic tap and water 
Citation: Umezawa K, Asai S, Iwashita H, Minakawa Y, Ohashi M, et al. (2016) Molecular Characteristics of a Series of Clinical Isolates of Drug-Resistant Acineto-

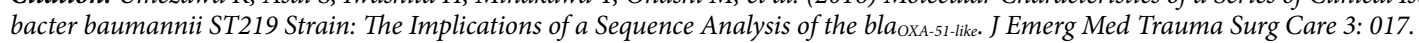

mixture side of the joint tube. Effective measures to minimize the risk in the wet environmental reservoir included strict sanitary management of the water systems in order to prevent future outbreaks. The MLST sequences between ST208 and ST219 differed by only a single $g p i$ base, and these isolates closely resembled one another in the molecular characteristics for resistance against each drug. This finding suggested that the ST208 and ST219 isolates were closely related in terms of genetics and might have been derived as subclones from the same origin.

In the detection of subsequent isolates, a reservoir was not identified despite environmental sampling for bacterial culture at several times. A more intensive environmental surveillance will be needed to identify the reservoir should DR-A. baumannii-2014 continue to be detected. However, the reinforcement of environmental disinfection, including clinical surfaces, and ensuring hand hygiene with alcohol containing antiseptic will be crucial for reducing the risk of cross-transmission in healthcare facilities [24].

In conclusion, a detailed molecular analysis of DR-A. baumannii would provide important knowledge for controlling nosocomial infections. Even when a different ST strain is detected, the sequencing of the $b l a_{\mathrm{OXA}-51 \text {-like }}$ would be useful for determining the clonality, which would thus make it possible to identify the relationship of A. baumannii infection.

\section{Acknowledgments}

This work was supported by JSPS KAKENHI Grant Numbers 25670768 and 26460657.

\section{References}

1. Dijkshoorn L, Nemec A, Seifert $H$ (2007) An increasing threat in hospitals: multidrug-resistant Acinetobacter baumannii. Nat Rev Microbiol 5: 939-951.

2. Asai S, Umezawa K, Iwashita H, Ohshima T, Ohashi M, et al. (2014) An out-

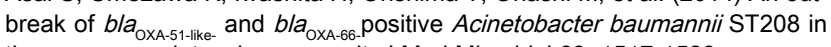
the emergency intensive care unit. J Med Microbiol 63: 1517-1523.

3. Umezawa K, Asai S, Ohshima T, Iwashita H, Ohashi M, et al. (2015) Outbreak of drug-resistant Acinetobacter baumannii ST219 caused by oral care using tap water from contaminated hand hygiene sinks as a reservoir. Am J Infect Control 43: 1249-1251.

4. Evans BA, Amyes SG (2014) OXA ß-lactamases. Clin Microbiol Rev 27: 241 263.

5. Poirel L, Naas T, Nordmann P (2010) Diversity, epidemiology, and genetics of class D beta-lactamases. Antimicrob Agents Chemother 54: 24-38.

6. Zander E, Nemec A, Seifert H, Higginsa PG (2012) Association between $\beta$-lactamase-encoding $b a_{\mathrm{OXA}-51}$ variants and DiversiLab rep-PCR-based typing of Acinetobacter baumannii isolates. J Clin Microbiol 50: 1900-1904.

7. Clinical and Laboratory Standards Institute (2010) Performance standards for antimicrobial susceptibility testing, 20th Informational supplement, CLSI, Wayne, PA.

8. Mohapatra BR, Broersma K, Mazumder A (2007) Comparison of five repPCR genomic fingerprinting methods for differentiation of fecal Escherichia coli from humans, poultry and wild birds. FEMS Microbiol Lett 277: 98-106.
9. Fu Y, Zhou J, Zhou H, Yang Q, Wei Z, et al. (2010) Wide dissemination of OXA-23-producing carbapenem-resistant Acinetobacter baumannii clonal complex 22 in multiple cities of China. J Antimicrob Chemother 65: 644-650.

10. Woodford N, Ellington MJ, Coelho JM, Turton JF, Ward ME, et al. (2006) Multiplex PCR for genes encoding prevalent OXA carbapenemases in Acinetobacterspp. Int J Antimicrob Agents 27: 351-353.

11. Turton JF, Ward ME, Woodford N, Kaufmann ME, Pike R, et al. (2006) The role of ISAba1 in expression of OXA carbapenemase genes in Acinetobacter baumannii. FEMS Microbiol Lett 258: 72-77.

12. Lee $Y$, Yum JH, Kim CK, Yong D, Jeon EH, et al. (2010) Role of OXA-23 and AdeABC efflux pump for acquiring carbapenem resistance in an Acinetobacter baumannii strain carrying the bla ${ }_{\mathrm{OXA}-66}$ gene. Ann Clin Lab Sci 40: 43-48.

13. Yamane K, Wachino J, Doi Y, Kurokawa H, Arakawa Y (2005) Global spread of multiple aminoglycoside resistance genes. Emerg Infect Dis 11: 951-953.

14. Endo S, Yano H, Hirakata Y, Arai K, Kanamori H, et al. (2012) Molecular epidemiology of carbapenem-non-susceptible Acinetobacter baumannii in Japan. J Antimicrob Chemother 67: 1623-1626.

15. Liu YH, Kuo SC, Lee YT, Chang, IC, Yang SP, et al. (2012) Amino acid substitutions of quinolone resistance determining regions in GyrA and ParC associated with quinolone resistance in Acinetobacter baumannii and Acinetobacter genomic species 13TU. J Microbiol Immunol Infect 45: 108-112.

16. Zander E, Chmielarczyk A, Heczko P, Seifert H, Higgins PG (2013) Conversion of OXA-66 into OXA-82 in clinical Acinetobacter baumannii isolates and association with altered carbapenem susceptibility. J Antimicrob Chemother 68: 308-311.

17. Fernando D, Kumar A (2012) Growth phase-dependent expression of RND efflux pump- and outer membrane porin-encoding genes in Acinetobacter baumannii ATCC 19606. J Antimicrob Chemother 67: 569-572.

18. Peleg AY, Seifert H, Paterson DL (2008) Acinetobacter baumannir: emergence of a successful pathogen. Clin Microbiol Rev 21: 538-582.

19. Hou PF, Chen XY, Yan GF, Wang YP, Ying CM (2012) Study of the correlation of imipenem resistance with efflux pumps AdeABC, AdelJK, AdeDE and AbeM in clinical isolates of Acinetobacter baumannii. Chemotherapy 58: 152-158.

20. Srinivasan VB, Rajamohan G, Pancholi P, Marcon M, Gebreyes WA (2011) Molecular cloning and functional characterization of two novel membrane fusion proteins in conferring antimicrobial resistance in Acinetobacter baumannii. J Antimicrob Chemother 66: 499-504.

21. Coyne S, Rosenfeld N, Lambert T, Courvalin P, Périchon B (2010) Overexpression of resistance-nodulation-cell division pump AdeFGH confers multidrug resistance in Acinetobacter baumannii. Antimicrob Agents Chemother 54: 4389-4393.

22. Rumbo C, Gato E, López M, Ruiz de Alegría C, Fernández-Cuenca F, et al. (2013) Contribution of efflux pumps, porins, and $\beta$-lactamases to multidrug resistance in clinical isolates of Acinetobacter baumannii. Antimicrob Agents Chemother 57: 5247-5257.

23. Dijkshoorn L, Nemec A, Seifert H (2007) An increasing threat in hospitals: multidrug-resistant Acinetobacter baumannii. Nat Rev Microbiol 5: 939-951.

24. WHO (2016) Clean Care is Safer Care. First Global Patient Safety Challenge, World Health Organization, Geneva, Switzerland. 